\title{
AJUSTES AMBIENTAIS NOS MODELOS DEA E A AGRICULTURA IRRIGADA
}

\author{
Yony SAMPaio * \\ Luciano Sampaio ${ }^{\dagger}$ \\ EMANOel de Souza Barros $\ddagger$
}

\begin{abstract}
Resumo
Modelos DEA têm sido extensivamente utilizados para medir eficiência e definir políticas. O modelo original não considera o caráter dinâmico e estocástico do ambiente agrícola. Em consequência, um produtor pode tomar decisões corretas mas obter um resultado menos satisfatório que outro devido a mudanças de preço ou de produtividade, decorrentes, por exemplo, do excesso de chuva. Para corrigir este problema é proposto um modelo em dois estágios que incorpora distribuições de preço e produtividade. Resultados de eficiência de produtores da fruticultura irrigada apontaram distribuição dos escores de eficiência do DEA-ajustado mais concentrada, suavizando os efeitos da aleatoriedade característica da produção agrícola.
\end{abstract}

Palavras-chave: Eficiência; Agricultura irrigada; Ajustes ambientais.

\begin{abstract}
DEA models are frequently used to measure efficiency and propose policies. The original model does not consider the dynamic and stochastic set of agricultural production. As a consequence, a producer can make the correct decisions but obtain less satisfactory results due to price or productivity changes, resulting, for example, from excessive rain. To correct this problem a two-stage model that consider price and productivity distributions is proposed. Results comparing the efficiency of irrigated agriculture producers show that the distribution of efficiency scores are more centered, reducing the stochastic effects typical of agricultural production.
\end{abstract}

Keywords: Efficiency; Irrigated agriculture; Environmental adjusts. JEL classification: Q10, Q12, C14

\footnotetext{
* Professor Titular do Departamento de Economia da UFPE. Email: yonysampaio@gmail.com

† Professor do Departamento de Administração da UFRN. Email: luciano.sampaio@pq.cnpq.br ‡ Professor do Departamento de Economia da UFPE, Campus Caruaru. Email: embarros2002@yahoo.fr
} 


\section{Introdução}

O trabalho seminal de Charnes et al. (1978) generalizou o uso, nas diversas áreas, dos modelos de Análise de Envoltória de Dados (DEA) para medir a eficiência. Na agricultura irrigada, entre as inúmeras aplicações, citam-se: Nogueira (1996), Conceição \& Araújo (2000), Mariano \& Sampaio (2002), Mariano (2004), Barros et al. (2004), Campos \& Ferreira Neto (2008), Mariano \& Pinheiro (2009). A eficiência pode ser obtida com a utilização de fronteiras paramétricas ou não paramétricas. Os modelos DEA estimam uma fronteira não paramétrica. Esses modelos tanto podem ser orientados pelo produto como pelo insumo e compreendem modelos com retornos constantes ou variáveis à escala. Neste artigo optou-se pelo trabalho com o modelo DEA orientado pelo produto e com retornos variáveis à escala. Mesmo nos modelos de fronteira estocástica (Aigner et al. 1977) o problema destacado neste trabalho persiste.

Um dos pressupostos, nem sempre observado nas análises, é o de homogeneidade da atividade exercida pelos produtores analisados. Na agricultura irrigada, esta questão é particularmente relevante dadas as rotação e diversidade de culturas adotadas pelos produtores. Algumas influências ambientais só afetam determinados produtos e, portanto, a diversidade dos mesmos, introduzindo um viés no cálculo da eficiência. A incorporação dessas influências ambientais nos modelos de eficiência, mais especificamente, nos modelos DEA, requer uma breve discussão dos conceitos e uma metodologia que permita corrigir as distorções introduzidas.

Nos modelos DEA, a fundamentação teórica é a mais simples possível: a eficiência, por parte do produtor ou do tomador de decisão, na escolha dos produtos e dos insumos de modo a obter um máximo de algum produto. A eficiência é caracterizada, de modo simples, como o produto máximo a ser obtido com uma dada quantidade de insumos ou como a mínima quantidade de insumos necessária para obter uma quantidade fixa de produto.

Ocorre que a teoria de produção há muito reconhece que o ambiente no qual a produção está inserida é dinâmico e estocástico, e que a produção obtida nem sempre corresponde ao planejado, devido à dada a existência de alguma aleatoriedade no processo de produção, que ocorre entre o momento da decisão do que produzir e o da obtenção do produto. São esses elementos não incorporados na análise de fronteiras eficientes que limitam os resultados obtidos e a extrapolação das conclusões a que esses trabalhos têm chegado. Caso a aleatoriedade seja comum a todas as atividades e/ou produtores, sua incorporação em modelos estocásticos ou mesmo o uso de bootstrap em modelos DEA atenua o problema. Se a influência do ambiente não é comum a todos os produtos, tem-se um viés no cálculo da eficiência. A volatilidade de preços, por exemplo, pode ocasionar grande alternância de receita para produtores com multiprodutos.

Os aperfeiçoamentos do modelo DEA que podem resolver, ao menos parcialmente, esses problemas, incluem avanços contidos em dois artigos de Sengupta, que primeiro incorporou a dinâmica Sengupta (1999) e depois adicionou a incerteza Sengupta (2005). Contudo estes não têm sido largamente usados, principalmente pela necessidade de dados de painel. A correção proposta neste artigo direciona-se para trabalhos baseados em dados cross-section, não havendo dados de painel para as unidades analisadas. Caso existam dados de painel é sugerido utilizar modelos DEA dinâmicos.

Em outra linha, há uma série de trabalhos que procuram corrigir a influên- 
cia do ambiente em modelos DEA aplicados ao transporte (Nolan 1996, Nolan et al. 2001, Odeck \& Alkadi 2001, Pina \& Torres 2001, Boame 2004, Odeck 2006, Barnum et al. 2007, 2008). No trabalho mais recente, Barnum et al. (2008) utilizam o método de estimação em dois estágios, por eles denominado de método de dois estágios reversos, no qual no primeiro estágio o produto é ajustado para as diferenças de ambiente, e no segundo, o modelo DEA é estimado com os insumos e esses produtos ajustados.

Este trabalho pretende delinear melhor as deficiências do método DEA original - na forma como é correntemente usado para análise de eficiência da agricultura irrigada - ocasionadas pela influência do ambiente e então propor um método em dois estágios. Por fim, na linha do que usa as aplicações de transporte citadas, comparando-se as medidas de eficiência do modelo proposto com correção do efeito ambiental às do modelo original em uma aplicação na agricultura irrigada.

Este artigo está dividido em três partes: a sessão seguinte descreve a eficiência no contexto da teoria econômica, mostrando suas limitações e distorções, distinguindo uma eficiência endógena, determinada exclusivamente pela empresa, de uma eficiência exógena, a qual sofre influências aleatórias. A seção 3 apresenta um modelo em dois estágios para corrigir as influências ambientais. A sessão 4 compara a eficiência da agricultura irrigada do Vale do São Francisco antes e após a correção para a influência do ambiente. Na seção 5, tem-se as conclusões.

\section{Eficiência Endógena e Exógena e os Efeitos Ambientais}

A eficiência pode ser definida como a escolha da melhor tecnologia e da combinação ótima de insumos e produtos que permitem alocar um dado produtor sobre a fronteira de produção. No entanto, o ambiente em que se dá a produção condiciona as variáveis econômicas. Os preços, por exemplo, apresentam variações sazonais e flutuações de prazo mais longo. Como o processo produtivo não é instantâneo, as condições ambientais podem variar entre um e outro momento. Ou, de modo mais simples, um produtor pode estabelecer um plano de produção que contemple uma combinação ótima de produtos e insumos e ver frustrado esse plano por mudanças no mercado e nos preços. A Figura 1 ilustra um produtor que seria eficiente no ponto A (Figura $1 \mathrm{~A}$ ) ou no ponto D (Figura $1 \mathrm{~B}$ ) com os preços vigentes no tempo $t_{0}$, mas que se torna ineficiente no tempo $t_{1}$.

Este exemplo mostra que pode-se definir uma eficiência endógena e uma eficiência exógena, a primeira sendo determinada exclusivamente pela empresa e a segunda pelo ambiente. Caso a influência do ambiente seja aleatória para todos os produtores, a eficiência relativa não se altera. Além disso, o produtor sabe que sua atividade é exercida em um ambiente dinâmico e de incerteza, e como consequência sua estratégia deve incorporar esses elementos. Mas, na agricultura irrigada, o efeito do ambiente pode ser assimétrico entre produtores. Mesmo admitindo que a atividade é profundamente homogênea e que o ambiente afeta igualmente a todos os produtores, há muitas outras variáveis intervindo no processo de produção que podem afetar diferencialmente os produtores. Assim, um produtor que tivesse errado seu plano produtivo, como em D (Figura $1 \mathrm{~B}$ ), acabaria, no tempo $t_{1}$, como mais eficiente, por acaso e não por uma decisão consciente. 


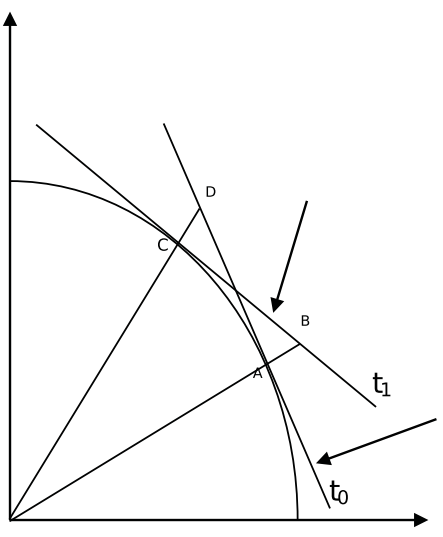

Figura $1 \mathrm{~A}$

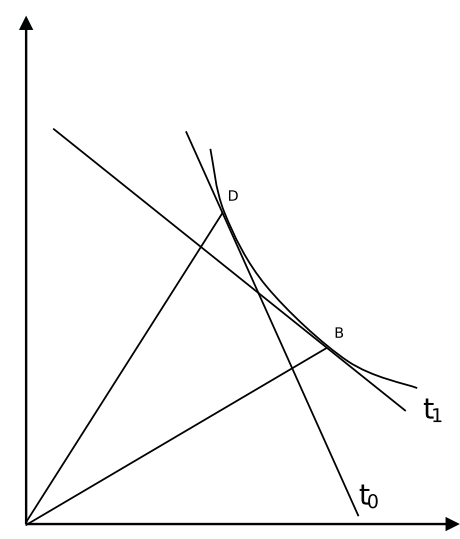

Figura 1B

Figura 1: Variação nos índices de preço entre o período $t_{0}$ e $t_{1}$ e sua influência sobre a eficiência

Pode-se argumentar que, ao decidirem um plano de produção, os produtores formulam expectativas quanto ao ambiente. Os clássicos modelos de expectativas adaptadas e ajuste parcial assumem que os produtores formulam expectativas (quanto ao preço, por exemplo) e ajustam sua produção em função da variação entre o evento e a expectativa ou em razão da sua capacidade de ajuste. Mas não é crível esperar que as expectativas formuladas se realizem com precisão nem parece correto dizer que o mais eficiente é aquele que foi mais bem sucedido em antecipar a direção das mudanças, em um único ano. Esse raciocínio só poderia ser válido se a eficiência fosse estimada para uma sucessão de períodos, podendo-se somar as eficiências e caracterizar a eficiência em um lapso maior de tempo. Mas este não é o procedimento usual na grande maioria dos trabalhos de fronteiras eficientes, os quais utilizam dados de corte transversal.

Dessa forma, a eficiência exógena (ou seja, com influências externas à empresa) é diferente da eficiência endógena (determinada assumindo que o ambiente não se altera, não há aleatoriedade nem variações temporais), mas a exógena é de fato a relevante para aferir a capacidade de o produtor escolher o plano de produção ótimo dadas as suas expectativas. Dada uma base de dados adequada, pode-se utilizar os modelos desenvolvidos por Sengupta (1999, 2005). Mas a prática usual, na literatura que mede a eficiência na agricultura irrigada, é tratar os produtores como exercendo uma atividade homogênea e utilizando dados de corte transversal, ou seja, assume-se que o ambiente ou não é importante ou que afeta igualmente todos os produtos e/ou produtores.

\subsection{Influências Aleatórias na Produção}

As alterações no processo de produção constituem um elemento de destaque implícito nas análises de eficiência. É claro que a escolha de uma tecnologia, por exemplo, condiciona os resultados. Assim, não é completamente ruim a ideia de tentar explicar a ineficiência estimada como decorrente, em parte, da adoção ou da escolha incorreta da tecnologia de produção. Ou seja, a eficiência endógena, determinada pelo produtor, não reflete a complexidade de sua 
inserção no mercado, a qual é captada pela eficiência exógena. Entretanto, há outros elementos envolvidos.

A produtividade pode se alterar em decorrência de fatores aleatórios, que afetam diferentemente cada produto e cada produtor. No caso da produção agrícola, o produtor pode planejar obter um determinado volume de produto e terminar com outro. Uma precaução é estimar fronteiras só para produtos homogêneos e assumir que as alterações afetem igual e indistintamente todos os produtores. Apesar da hipótese ser heroica, o resultado, ainda assim, turva a análise de eficiência. O que seria eficiente deixou de ser, porque usou insumos em demasia para uma produção diminuída, e o que seria menos eficiente passou a ser eficiente porque usou poucos insumos, teve a produção diminuída, mas o retorno caiu menos que proporcionalmente. Caso a produção seja heterogênea ou múltipla, como na agricultura irrigada, mas também para serviços bancários, médicos, educacionais, administração pública, as consequências podem ser piores. A Figura 2 ilustra bem o caso de dois produtores e um insumo, com alterações na produtividade.

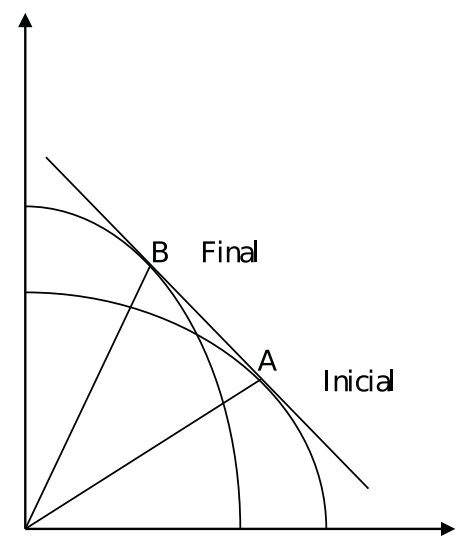

Figura $2 \mathrm{~A}$

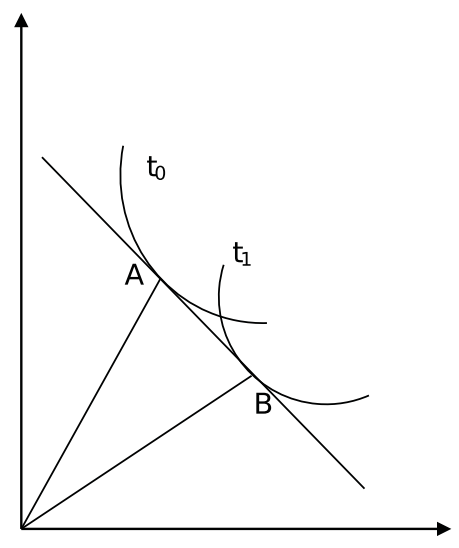

Figura 2B

Figura 2: Variação na produtividade e seu efeito sobre a eficiência

Dadas as condições iniciais de produção, o produtor em A seria mais eficiente. Mas, dada a alteração de produtividade, o produtor $B$, ineficiente inicialmente, torna-se eficiente.

A solução desse problema é deveras complicada, pois depende de uma série de dados particulares a cada produtor. Diferentemente de mudanças ambientais, como preços, que afetam todos mas não dependem de cada produtor individualmente (assumindo-se um grau razoável de competição), as alterações na produtividade tanto refletem mudanças ambientais como alterações no plano de produção. Como é impossível isolar essas mudanças e alterações, seria necessário tomar dados individualizados e estimar uma sequência de fronteiras obtendo-se, novamente, uma distribuição de eficiência para cada produtor. O recurso à média nesae caso não apresenta resultados satisfatórios, pois elimina um fator distintivo entre produtores: o plano de produção. 


\subsection{Heterogeneidade do Conjunto de Produtos}

A ideia por traz do modelo DEA estocástico é que, para cada tempo t, é possível obter uma distribuição de eficiência e assim estimar os escores de eficiência com base nessa distribuição.

Um caso mais grave ocorre quando a analise é aplicada a uma região, supostamente homogênea, mas na qual, como quase sempre ocorre na agricultura, os produtores diversificam um pouco a produção.

A equação de lucro:

$$
\Pi=\sum_{i} p_{i} y_{A_{i}}-\sum_{j} r_{j} X_{j}
$$

onde $p_{i}$ é o preço por unidade de produto, $y_{i}$ a produtividade por área e $A_{i}$ é a área utilizada, $r_{j}$ e $X_{j}$ são os preços e quantidades de insumos, respectivamente, que mostram que as alterações na produtividade e nos preços podem induzir grandes alterações de eficiência entre anos. O problema é particularmente grave se essas alterações não forem homogêneas entre produtos: por exemplo, as plantas são mais ou menos resistentes à chuva e à seca; o vento e a geada alteram cada cultura de modo distinto; o custo de produção depende mais ou menos do preço do petróleo. Algumas dessas influências externas afetam tão fortemente a produção e o seu valor que geram um conjunto de eficiências tão particulares que distorcem completamente as possíveis inferências sobre produtores eficientes e ineficientes.

Algum ajuste é possível neste caso para compensar as alterações nos preços e na produtividade. Pode-se ajustar o preço informado por cada produtor pela diferença entre o preço anual médio em um período de anos e o preço médio anotado naquele ano. Dessa forma, corrigem-se as variações não previstas no preço, mas mantêm-se as variações decorrentes das estratégias de venda e da variação de qualidade do produto, particulares a cada produtor. $\mathrm{O}$ ajuste da produtividade é mais problemático, pois assume que as condições de produção se mantêm inalteradas ao longo do período considerado, variando a produtividade apenas em função dos elementos aleatórios. É claro que, se há alterações mensuráveis nas condições de produção, estas podem ser levadas em conta, mas dificilmente este será o caso.

Há ainda a alternativa de serem geradas distribuições distintas para o preço e a produtividade, obtendo-se uma distribuição para cada produtor, mas o recurso do ajuste em função da média é bem mais simples e resulta em uma única distribuição para todos os produtores.

\section{Correção para Influências Ambientais - o Modelo de Dois Estágios}

Na agricultura, a produção é, de modo geral, diversificada, variando dentro de um mesmo ano agrícola e entre anos, e está baseada na utilização de técnicas modernas de irrigação, adaptadas a cada tipo de cultura. Os produtores agrícolas produzem uma ou mais culturas, gerando uma produção heterogênea e que não pode ser mensurada de forma agregada, em volume. Mesmo que tais produções sejam agregadas em valores monetários, a aleatoriedade de preço e produtividade, entre culturas, não é detectada pelos modelos clássicos de estimação de fronteiras de produção. 
Alguns produtores podem ter alocado corretamente os insumos disponíveis na produção de suas culturas, mas fatores aleatórios (como vento, inundações, etc.) impossibilitaram a obtenção de uma produção ótima em uma delas, colocando-a como ineficiente. Na verdade, ele planejou corretamente a produção de cada uma de suas culturas e, pela receita esperada, deveria estar entre os mais eficientes. Mas devido a um vento ou outro fator aleatório, boa parte da produção de uma cultura em particular (sujeita ao efeito dos ventos) foi perdida. Note que o complicador principal decorre da produção ser heterogênea e da influência ambiental não ser homogênea entre culturas.

Outro fator importante que afeta a eficiência é a variação do preço do produto. O produtor pode ter planejado sua produção com base nos valores de receitas obtidos em anos anteriores, mas, devido a algum fator aleatório, o preço do produto pode sofrer variações. No caso de culturas heterogêneas, o preço de uma das culturas pode ter sofrido aumento, modificando assim o equilíbrio ótimo para esta cultura, e, de forma indireta, alocando o produtor como ineficiente em relação à fronteira ótima. Novamente, caso houvesse homogeneidade de produto, o efeito seria igual para todos e não causaria distorções; não sendo, pode ocorrer introdução de viés.

Pode-se introduzir igualmente a correção no preço dos insumos, mas esses preços apresentam menor variação entre produtores e em um período de tempo. Além disso, por compreender muitos insumos, geralmente agregados em valor, não possuem séries de preço divulgadas.

Para corrigir tais problemas, propõe-se um modelo de dois estágios que leve em conta as variações no preço e na produtividade, mesmo na presença de culturas heterogêneas. O modelo aqui proposto assemelha-se ao utilizado por Barnum et al. (2008), para corrigir diferenças de fluxo em linhas de transporte urbano. No primeiro estágio é feita a correção do ambiente no preço do produto e na produtividade.

Para calcular o preço corrigido, usa-se a seguinte expressão:

$$
\hat{P}_{i j_{t}}=\frac{V B P_{i j_{t}}}{Q_{i j_{t}}}
$$

onde $P_{i j_{t}}, V B P_{i j_{t}}$ e $Q_{i j_{t}}$ representa o preço da cultura $i$, o valor bruto da produção e a quantidade produzida da cultura $i$, todos referentes ao projeto de irrigação $j$ no tempo $t$. Obtidos os preços médios para uma série de anos, é calculada a média dos preços, sendo T o total de observações:

$$
\overline{\hat{P}_{i j}}=\sum_{t} \frac{\hat{P}_{i j_{t}}}{T}
$$

Comparando com o preço médio de um ano particular, , obtém-se a variação anual de preço

$$
\Delta P_{i j_{t}}=\overline{\hat{P}_{i j}}-\hat{P}_{i j_{t}}
$$

Tomando-se esta variação em percentagem, o preço recebido por cada produtor, em um dado ano, é reajustado. A suposição, é que a aleatoriedade, no caso o mercado, afeta igualmente todos os produtores. O resultado é obter uma distribuição ajustada em procedimento similar ao utilizado em modelos nos quais se retira o efeito da sazonalidade, só que, no caso, as influências ambientais são diferentes para cada produto. 
Um procedimento idêntico é adotado para recalcular a produtividade obtida por cada produtor, em um projeto de irrigação $j$. Espera-se através deste procedimento obter um índice de preço, uma produção e uma renda confiáveis por cultura de modo a compará-los aos valores fornecidos pelos colonos. A separação desses índices por projeto de irrigação é feita para dar mais consistência aos valores corrigidos, uma vez que os colonos de um mesmo projeto possuem, em hipótese, características semelhantes e encaram preços e produção de forma idêntica.

A quantidade produzida por cultura é obtida através do índice de produtividade para os projetos de irrigação do polo. De maneira formal, esse índice pode ser calculado pela expressão:

$$
\hat{Y}_{i j}=y_{i j} * A C_{i j}
$$

onde $y_{i j}$ e $A C_{i j}$ representa a produtividade e a área colhida da cultura $i$ no projeto de irrigação $j$.

O valor da produção (ou receita) para um dado produtor $r$ inserido em um dado projeto de irrigação $j$ será então:

$$
V \hat{P}_{r}=\sum_{i j} \hat{P}_{i j} * \hat{Y}_{i j}
$$

onde $V \hat{P}_{r}$ representa a receita obtida pelo produtor $r$ através da comercialização de todas as culturas que ele produz utilizando a tecnologia do projeto de irrigação $j$.

Caso se deseje estimar uma fronteira de produção, e tomando o Valor da Produção (VP), ou receita, como uma proxy da quantidade produzida, este modelo terá o formato:

$$
V P^{*}=f(I N, K, M O)
$$

onde $V P^{*}$ representa o vetor $[n \times 1]$ de todas as $n$ observações de produtores do polo irrigado. IN, $K$ e $M O$ descrevem a matriz das variáveis explicativas formada, neste exemplo, pelos gastos com insumos, capital disponível e mãode-obra, respectivamente.

O segundo estágio consiste em estimar modelo DEA-V com os produtos corrigidos, adotando-se a orientação pelo produto. A escolha do modelo orientado pelo produto é decorrida do fato de que na produção agrícola, em geral, pretende-se maximizar o produto obtido dados os insumos utilizados. Adotou-se ainda a fronteira com retorno variável à escala por esta se adaptar melhor à diversidade da agricultura irrigada.

$$
\begin{aligned}
& \max _{\theta, \lambda} \theta^{*} \\
& \text { s.t. } X \lambda \leq X_{0} \\
& \theta V P^{*}{ }_{0}-V P^{*} \lambda \leq 0 \\
& \lambda \geq 0, e^{T} \lambda=1
\end{aligned}
$$

onde $X$ representa a matriz de variáveis explicativa, $e^{T}$ é um vetor linha unitário e $\lambda$ o peso de cada produtor no processo produtivo. 
As restrições deste modelo levam em conta os valores corrigidos para cada cultura em termos de receita (VP) e os agrega em nível de produtor. O nível de eficiência será dado pela inversa da solução do problema de maximização $\frac{1}{\theta^{*}}$.

Se $\hat{P}_{i j} \cong P_{i j}$ e $\hat{Y}_{i j} \cong Y_{i j}$, os preços e produtos corrigidos serão aproximadamente iguais aos valores fornecidos pelos produtores. Os níveis de eficiência serão próximos dos valores estimados pelo modelo DEA-V e a influência de fatores aleatórios, não quantificáveis por este modelo, seria, nesse caso, mínima. Caso contrário, os níveis de eficiência estimados pelo modelo de dois estágios, simplificadamente chamado de modelo DEA-V ajustado, serão mais consistentes que os estimados pelo modelo DEA-V padrão. Os índices corrigidos estariam, nesse caso, "amortizando" o efeito da influência do ambiente no processo produtivo e quantificando índices de eficiência ligados unicamente à alocação dos recursos na produção determinada pela escolha dos produtores e uma aleatoriedade comum.

\section{Comparando a Eficiência da Agricultura em Perímetros Irrigados do Sub-Médio São Francisco}

\subsection{Análise descritiva dos dados}

Os dados utilizados foram coletados para a pesquisa Investimento Público e Privado em Agricultura Irrigada e seus Determinantes sobre o Emprego e a Renda, aplicada entre março e setembro de 1998 (FADE 1998, Sampaio \& Sampaio 2004).

Dada a complexidade da base de dados e para extrair somente os dados que serão analisados neste artigo, foram selecionados apenas os referentes aos colonos produtores de fruticultura irrigada, mais precisamente os produtores de uva, manga, banana, coco, goiaba e acerola inseridos nos projetos Bebedouro, Maniçoba, Curaça, Tourão e Nilo Coelho. Estas são culturas mais amplamente adotadas e para as quais existe um número maior de observações.

A estimação das fronteiras de produção é efetuada com a agregação de todos os produtos por colono, contemplando um total de 149 colonos especializados na produção dos seis tipos de culturas citadas acima. Dada essa diversidade, o modelo de retornos variáveis é mais adequado. A variável dependent será - Valor da Produção - considerada uma proxy da quantidade produzida. As variáveis exógenas são: área colhida, em hectare, que descreve a área na qual foi obtida a produção das culturas supracitadas; despesa com insumos, em dólar (US\$), que descreve o total das despesas com sementes, mudas, adubos, herbicidas, combustíveis, água e energia; capital, em dólar (US\$), que descreve o inventário dos prédios e equipamentos presentes na propriedade do colono; e, mão-de-obra, em horas/ano, que corresponde à mão-de-obra familiar ou contratada pelo colono no processo de produção, comercialização e transporte das culturas irrigadas.

A estatística descritiva dos dados (Tabela 1) mostra que as variáveis "valor da produção", "área colhida", "despesa com insumos", "capital" e "mãode-obra" possuem índices médios de US $\$ 10.753,62 ; 4,43$ ha; US $\$ 2.579,61$ e US\$8.812,55 e 295, respectivamente. Todavia, o desvio padrão é relativamente grande. A variação decorre tanto da combinação de culturas, com exigências técnicas distintas, como da variação da área média, além do efeito decorrente do processo de decisão de cada produtor. 
Tabela 1: Estatística descritiva dos dados

\begin{tabular}{llrrrr}
\hline Variáveis & $\begin{array}{l}\text { Unidade de } \\
\text { medida }\end{array}$ & Média & Desvio-padrão & Mínimo & Máximo \\
& & & & \\
\hline Valor da produção & Dólar (US\$) & $10.753,62$ & $16.686,16$ & 150,00 & $120.000,00$ \\
$\begin{array}{l}\text { Valor da produção } \\
\text { (modelo corrigido) }\end{array}$ & Dólar (US\$) & $17.368,97$ & $11.575,16$ & 437,72 & $73.410,86$ \\
Área irrigada & Hectare & & & & \\
Insumos & Dólar (US\$) & $2.579,61$ & $2.274,69$ & 238,00 & $12.955,00$ \\
Capital & Dólar (US\$) & $8.812,55$ & $10.506,28$ & 400,00 & $76.600,00$ \\
Mão-de-obra & Horas/ano & 295,00 & 209,66 & 12,00 & $1.210,00$ \\
\hline
\end{tabular}

Fonte: FADE (1998) e realização própria.

O valor da produção corrigido pelas equações (2) a (6) possui média um pouco mais elevada (US\$17.368,97), mas com um desvio-padrão proporcionalmente menor que o apresentado pela base de dados original. Isso significa que os valores estão mais centrados em torno da média. Os valores mínimos e máximos, variaram de $U S \$ 150,00$ a $U S \$ 437,72$, e de $U S \$ 73.410,86$ para US\$120.000,00, respectivamente. O maior valor de produção encontrado pelo modelo corrigido é quase duas vezes menor que o fornecido pela base de dados original. A primeira conclusão a que se chega, ao comparar esses valores, é que parte da variação encontrada na base de dados original parece ser decorrente das influências ambientais afetando a produtividade. ${ }^{1} \mathrm{O}$ cálculo de eficiência, com base na mesma, é enganoso, pois premia como mais eficientes os que apenas foram beneficiados pelo acaso e condena como ineficientes os que, apesar de tomarem decisões produtivas corretas, foram prejudicados pelo acaso.

É necessário salientar que alguns colonos são especialistas na produção de dois ou três tipos de culturas, enquanto que outros se concentram na produção de uma única cultura. Isso gera diferenças entre colonos no tocante ao nível de despesas com insumos, capital e mão-de-obra, que são proporcionais às áreas dedicadas a cada cultura.

Dos 149 colonos de toda a amostra, 75 (50,33\%) produzem unicamente banana ou uma combinação de banana com outra cultura. Destes 75 produtores de banana, 66 colonos ( $88 \%$ deste grupo) estão inseridos no projeto Nilo Coelho.

Do total de produtores inseridos no Nilo Coelho, os que estão produzindo banana, o fazem, ou como cultura de transição (juntamente com outras culturas), ou como cultura principal. Dos $6.548,50$ ha cultivados pelas seis culturas analisadas neste trabalho, 2.852, 40 ha são utilizados somente na produção de banana, gerando uma produção de 80.000 toneladas com renda estimada em 27 milhões de dólares ( $45 \%$ da renda bruta total). Isso mostra o impacto que a cultura de banana tem no projeto no ano em análise. Ocorre que no ano em que os dados foram coletados ocorreram fortes ventos que derrubaram muitas bananeiras fazendo com que a produtividade fosse bem mais baixa que a usual. A influência do vento sobre as outras culturas foi muito pouco relevante. O cálculo de eficiência sem levar em conta este fato se apresenta bastante distorcido.

\footnotetext{
${ }^{1}$ Esta variação pode ser devida a diversas causas, mas uma das principais decorre da variação de produtividade entre anos que, no caso da agricultura, deve-se principalmente a variações climáticas que afetam distintamente as culturas, ainda que com irrigação.
} 


\subsection{Comparando a Eficiência padrão com a ajustada pelos fatores ambientais}

Para a análise das distorções do ambiente, no caso, principalmente o vento, em modelos DEA, foram estimados o modelo DEA-V com a utilização da base de dados original e o modelo de dois estágios, aqui chamado de DEA-V ajustado, no qual foram feitas correções para a influência do ambiente introduzindo a distribuição de preço e produtividade para cada uma das seis culturas e para cada um dos seis perímetros irrigados.

No tocante à distribuição dos níveis de eficiência de ambos os modelos, pode-se observar que o modelo ajustado elevou os níveis de eficiência de forma significativa. A eficiência média praticamente dobrou, passando de 0,315 para 0,556. Consequentemente, a distribuição dos níveis de eficiência seguiu este movimento "para cima", deslocando-se para níveis mais próximos à unidade (eficiência plena). Este aumento da eficiência média é esperado pois está sendo eliminada uma fonte de variação.

A Figura A.1 mostra as distribuições de ambos os modelos. No caso do modelo DEA-V, a maior parte dos escores de eficiência se concentra nos pontos extremos do gráfico, entre $0-0,4$ e $0,9-1$. As variações no preço e na produtividade ao longo do tempo geram este tipo de problema, uma vez que são tidas pelo modelo como "má alocação de recursos", puxando os níveis de eficiência para baixo ou para cima (no caso dos produtores eficientes por acaso). Assim, a distribuição parece concentrada em intervalos extremos.

Ao se analisar a distribuição do modelo DEA-V ajustado, pode-se constatar que os níveis estão mais concentrados nos intervalos $0,2-0,4$ e $0,5-0,8$, efeito este devido à correção no problema das variações dos preços e produtividades e também do aumento da eficiência média. Isso se deve também ao fato do modelo corrigido uniformizar a diferença entre projetos de irrigação e produtores de forma simultânea. Interessantemente, a eficiência média é elevada, mas o número de DMUs eficientes é menor no DEA-V ajustado.

\subsection{Comparação por atividade / produto}

As Figuras A.2 e A.3 descrevem as distribuições de eficiência para as culturas de uva, manga, banana, coco, goiaba e acerola.

As distribuições à esquerda descrevem as distribuições do modelo DEA-V e as da direita, as distribuições estimadas pelo modelo DEA-V ajustado. As culturas de uva e manga não apresentam grandes variações quando submetidas ao DEA-V ajustado. Ou seja, as informações fornecidas pelos colonos produtores dessas culturas, no tocante à utilização dos recursos disponíveis, coincidiram com o nível de produção esperado pelos mesmos. Pode-se supor aqui que não houve "imprevistos" durante o processo produtivo de tais culturas.

No entanto, a cultura de banana apresenta grande variação quando submetida ao DEA-V ajustado. Os níveis de eficiência, que antes se concentravam no intervalo de 0 a 0,2, passaram se localizar no intervalo 0,4 a 0,8, uma variação bastante considerável para o contexto dos produtores de banana. A eficiência média mudou de 0,19 para 0,62. De fato, a cultura de banana enfrentou sérias perdas em termos de produtividade no ano agrícola de 1997, devido ao forte vento que derrubou muitos cultivares, fazendo com que o nível de produção esperado reduzisse aproximadamente à metade do esperado. 
Esse vento inesperado, fez com que os produtores de banana se apresentassem como ineficientes.

O modelo DEA-V ajustado mostra que os produtores de banana alocaram seus recursos de forma mais eficiente que a estimada sem correção, mas que devido ao "imprevisto" ocasionado pelo vento, seus níveis de eficiência foram deslocados para baixo. Pode-se observar também que certos produtores, que no modelo DEA-V estavam com níveis de eficiência próximos da unidade foram deslocados para escalas de eficiência mais baixa. Eles podem ter alocado os recursos de forma menos eficiente que os demais, mas devido ao vento e aos níveis de produção reduzidos a patamares inferiores, foram contados entre os eficientes, contudo eficientes por acaso.

A Figura A.3 mostra as distribuições de eficiência para as culturas de coco, goiaba e acerola. A cultura de coco manteve os níveis de eficiência situados no intervalo de 0 a 0,2 , mesmo após a simulação gerada pelo DEA-V ajustado. No entanto, a distribuição se apresentou mais uniformemente distribuída. As culturas de goiaba e acerola apresentaram deslocamentos para cima quanto aos níveis de eficiência, passando de 0 a 0,4 para 0,2 a 0,8 no caso da goiaba, e de 0 a 0,4 para 0,4 a 0,8 no caso da acerola. O efeito gerado pelo modelo DEA-V corrigido foi ainda inferior ao apresentado pela cultura de banana, mas pode-se também indicar má alocação de recursos em conjunto com fatores aleatórios, não quantificados pelo modelo padrão.

\section{Conclusões}

O artigo tem o propósito principal de mostrar como o modelo DEA é bastante suscetível às variações nos preços e na produtividade em análises de eficiência da agricultura irrigada. Sem ter como identificar tais variações de forma isolada, o modelo considera como ineficientes os produtores que provavelmente alocaram de forma correta os fatores de produção disponíveis, mas que devido a alguns imprevistos (como o vento que prejudicou a produção de banana) foram contados entre os ineficientes.

A análise de eficiência de acordo com a teoria, deve considerar, o mais possível, a escolha do produtor - a eficiência técnica e econômica na escolha de produtos e insumos, em processo estocástico e dinâmico. Mas os estudos usuais tomam dados de corte transversal e, ao não considerar a influência do ambiente, acabam calculando eficiências onde o acaso pode desempenhar papel importante. É claro que sugestões para melhoria da eficiência baseadas em modelo sem correções, além de fazer pouco sentido podem até induzir à ineficiência. No caso específico, por exemplo, uma recomendação errônea seria a substituição da cultura da banana.

Para superar esse problema, sugere-se a utilização do modelo DEA dinâmico, no sentido de calcular a eficiência ao longo do tempo, e estocástico, ao tomar a distribuição das variáveis pertinentes. Caso não exista uma série temporal, para todas as variáveis, ainda assim é possível a correção de algumas variáveis. No caso foi estimado um modelo de dois estágios no qual no primeiro estágio foi procedida correção pela série de preços e de produtividades médios, no qual os dados de cada DMU foram ajustados e no segundo estágio foi estimado o modelo DEA-V, aqui chamado de ajustado.

Comparando os modelo, constata-se que a eficiência média quase dobrou em relação ao modelo sem correção e a distribuição de eficiência ficou mais 
concentrada em torno da nova média e não em pontos extremos. Ou seja, corrigida a influência ambiental, constata-se que o processo de decisão dos produtores é mais homogêneo.

Diante desses aspectos, o modelo ajustado mostrou-se bastante útil na quantificação e identificação dos efeitos (ou vieses) decorrentes da variação dos preços e da produtividade, separando-a do processo de estimação dos escores de eficiência do modelo DEA. O modelo de dois estágios vem dar um suporte a mais às estimações de fronteiras de produção, uma vez que os efeitos ambientais (que não são quantificados diretamente pelo modelo) podem ser reduzidos, não sendo incorporados "erroneamente" aos níveis de eficiência. Assim, tem-se escores de eficiência mais consistentes e confiáveis, refletindo quase unicamente a alocação dos fatores de produção planejada pelos produtores e a aleatoriedade comum a todos. Essa formulação aproxima a estimação da concepção teórica de fronteira eficiente de produção.

\section{Referências Bibliográficas}

Aigner, D., Lovell, C. \& Schmidt, P. (1977), 'Formulation and estimation of stochastic frontier production function models', Journal of econometrics 6(1), 21-37.

Barnum, D., McNeil, S. \& Hart, J. (2007), 'Comparing the efficiency of public transportation subunits using data envelopment analysis', Journal of Public Transportation 10(2), 1-16.

Barnum, D., Tandon, S. \& McNeil, S. (2008), 'Comparing the performance of bus routes after adjusting for the environment using data envelopment analysis', Journal of Transportation Engineering 134(2), 77-85.

Barros, E., Costa, E. \& Sampaio, Y. (2004), 'Análise de eficiência estimando fronteiras paramétricas cobb-douglas e translog: o caso das empresas agrícolas do pólo petrolina-juazeiro', Revista Econômica do Nordeste 35(1), 7-19.

Boame, A. (2004), 'The technical efficiency of canadian urban transit systems', Transportation Research Part E: Logistics and Transportation Review 40(5), 401-416.

Campos, S. \& Ferreira Neto, J. (2008), 'Eficiência técnica dos produtores de leite em assentamentos rurais da reforma agrária', Revista Economia e Agronegócio 6(3), 395-414.

Charnes, A., Cooper, W. \& Rhodes, E. (1978), 'Measuring the efficiency of decision making units', European journal of operational research 2(6), 429-444.

Conceição, J. \& Araújo, P. (2000), 'Fronteira de produção estocástica e eficiência técnica na agricultura', Revista de Economia e Sociologia Rural 38(1), 4564.

FADE (1998), Investimento público e privado em agricultura irrigada e seus determinantes sobre o emprego e a renda, Technical report, FADE.

Mariano, J. (2004), Ineficiência técnica e desperdício da água na fruticultura irrigada no vale do são francisco, in E. Sampaio, ed., 'Ensaios sobre a economia da agricultura irrigada', Banco do Nordeste, Fortaleza, p. 236. 
Mariano, J. \& Pinheiro, G. (2009), 'Eficiência técnica da agricultura familiar no projeto de irrigação do baixo açu (rn)', Revista Econômica do Nordeste 40(2), 283-296.

Mariano, J. \& Sampaio, Y. (2002), 'A eficiência técnica dos colonos na agricultura irrigada no vale do são francisco', Economia Aplicada 6(2), 265-285.

Nogueira, M. (1996), Eficiência técnica na agropecuária das microrregiões Brasileiras, PhD thesis, Universidade Federal de Viçosa.

Nolan, J. (1996), 'Determinants of productive efficiency in urban transit', Transportation Research Part E: Logistics and Transportation Review 32(3), 319342.

Nolan, J., Ritchie, P. \& Rowcroft, J. (2001), 'Measuring efficiency in the public sector using nonparametric frontier estimators: a study of transit agencies in the usa', Applied Economics 33(7), 913-922.

Odeck, J. (2006), 'Congestion, ownership, region of operation, and scale: Their impact on bus operator performance in norway', Socio-Economic Planning Sciences 40(1), 52-69.

Odeck, J. \& Alkadi, A. (2001), 'Evaluating efficiency in the norwegian bus industry using data envelopment analysis', Transportation 28(3), 211-232.

Pina, V. \& Torres, L. (2001), 'Analysis of the efficiency of local government services delivery. an application to urban public transport', Transportation Research Part A: Policy and Practice 35(10), 929-944.

Sampaio, Y. \& Sampaio, E. (2004), Ensaios sobre a economia da agricultura irrigada, Banco do Nordeste do Brasil, Fortaleza.

Sengupta, J. (1999), 'A dynamic efficiency model using data envelopment analysis', International Journal of Production Economics 62(3), 209-218.

Sengupta, J. (2005), 'Nonparametric efficiency analysis under uncertainty using data envelopment analysis', International Journal of Production Economics 95(1), 39-49. 
Apêndice A
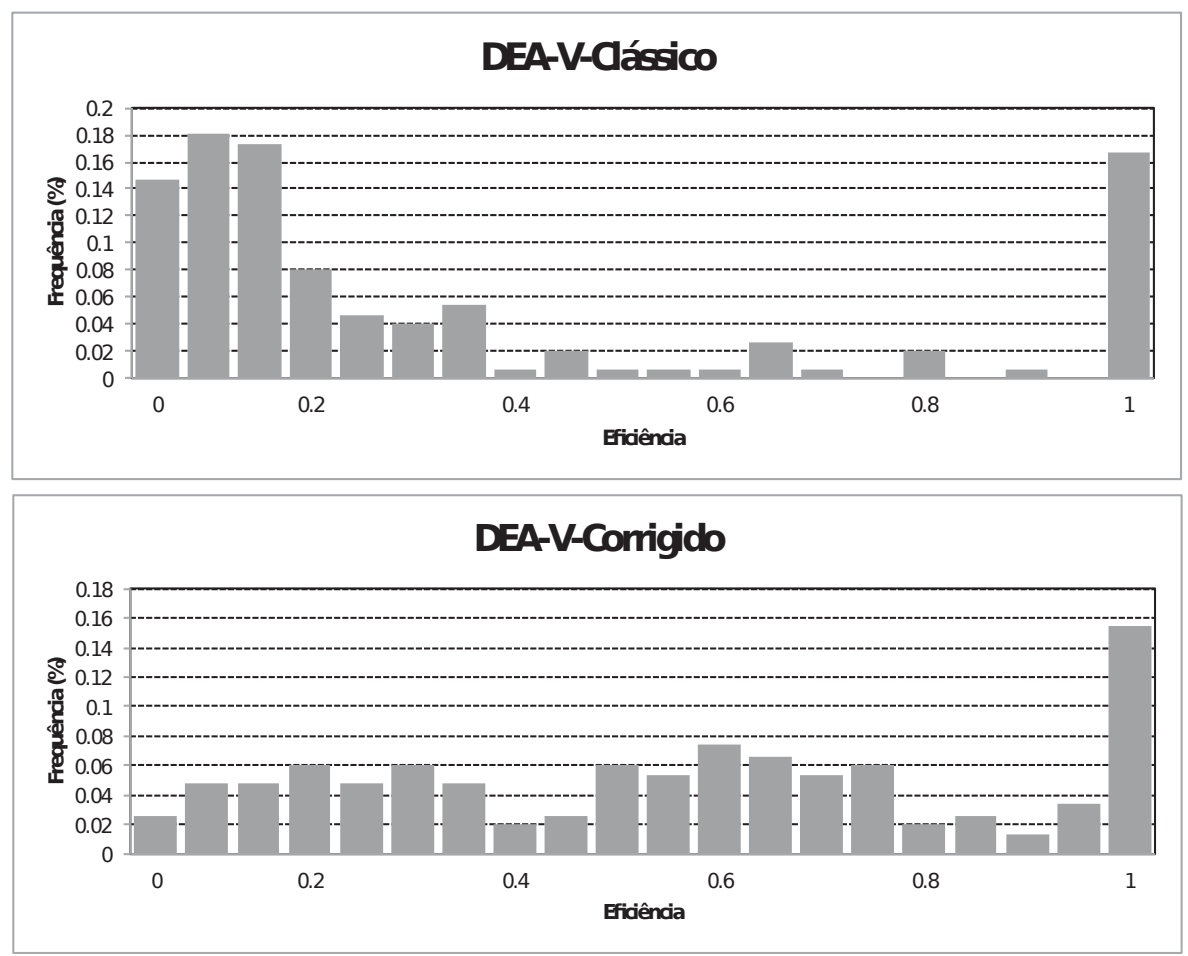

Figura A.1: Distribuição de eficiência dos modelos DEA-V-padrão e DEAV-ajustado 

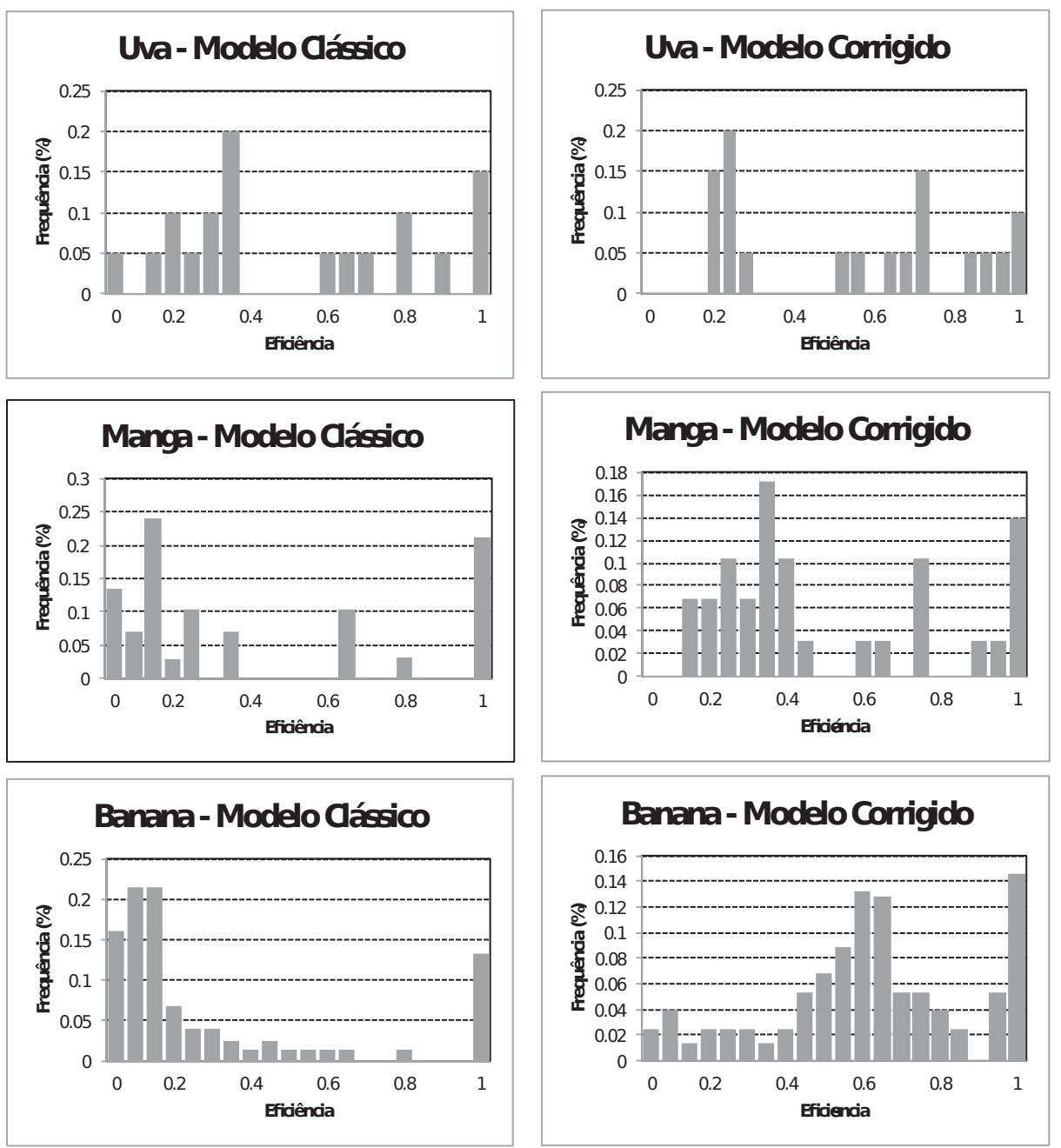

Figura A.2: Distribuição de eficiência dos modelos DEA-V-padrão e DEA-Vajustado para as culturas de uva, manga e banana 

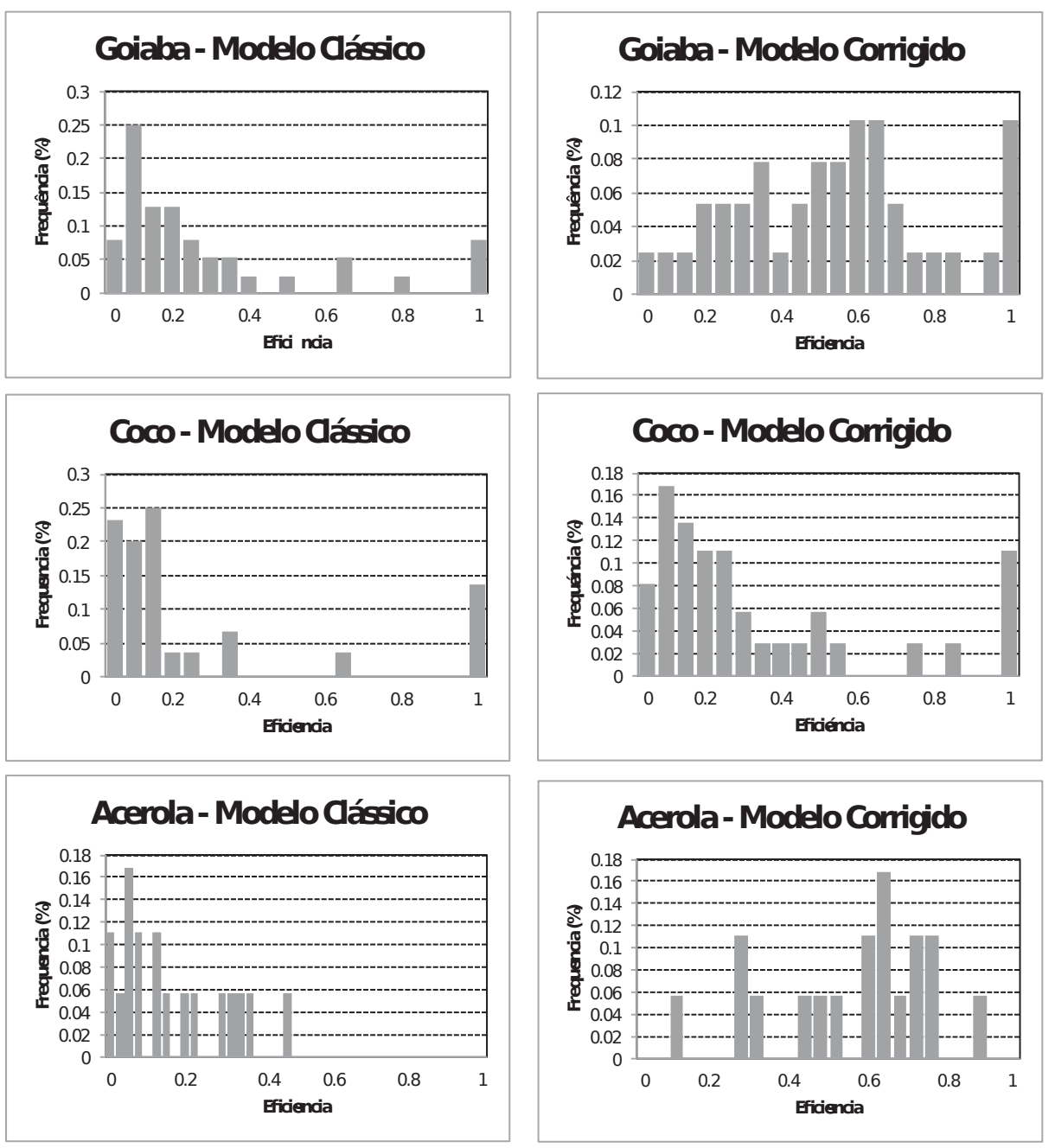

Figura A.3: Distribuição de eficiência dos modelos DEA-V-padrão e DEA-Vajustado para as culturas de goiaba, coco e acerola 